\title{
Eficácia de misturas formuladas contendo halauxifen-methyl no controle de soja voluntária DAS-444Ø6-6 (Enlist E3 ${ }^{\mathrm{TM}}$ )
}

\author{
Efficacy of halauxifen-methyl formulations to control volunteer soybean \\ DAS-444Ø6-6 (Enlist E3 ${ }^{T M}$ )
}

\author{
Luiz Henrique Saes Zobiole ${ }^{1 *}$, Kalsing Augusto ${ }^{1}$
}

Resumo - O controle da soja voluntária é primordial, principalmente pela presença de inóculos da ferrugem asiática nestas plantas. Com a introdução da soja tolerante ao 2,4-D (DAS-444Ø6-6 - Enlist E3 ${ }^{\mathrm{TM}}$ ), estudos são necessários para confirmar seu controle químico. O objetivo deste trabalho foi avaliar a eficácia no controle da soja voluntária DAS-444Ø6-6, utilizando herbicidas alternativos ao 2,4-D. O experimento foi realizado em condições de campo, no município de Cascavel, utilizando o delineamento em blocos ao acaso, com três repetições. Os tratamentos avaliados foram: halauxifen-methyl + pyroxsulam $\left(5+18\right.$ g i.a. ha $\left.{ }^{-1}\right)$, halauxifen-methyl + diclosulam $\left(5+25\right.$ g i.a. ha $\left.{ }^{-1}\right)$ e halauxifen-methyl +2,4-D $\left(5+783\right.$ g i.a. ha $\left.{ }^{-1}\right)$, o tratamento padrão paraquat $\left(400 \mathrm{~g}\right.$ i.a. ha $\left.\mathrm{a}^{-1}\right) \mathrm{e}$ uma testemunha sem aplicação de herbicidas. As aplicações foram realizadas em pós emergência da soja no estádio fenológico $\mathrm{R}_{2}$. Os resultados mostraram que aos 21 dias após aplicação todos os tratamentos apresentaram controle acima de $90 \%$ no controle da soja voluntária. As novas misturas formuladas contendo o herbicida halauxifen-methyl, foram eficientes no controle da soja voluntária DAS-444Ø6-6, em estádio fenológico avançado $\left(\mathrm{R}_{2}\right)$.

Palavras-chave: controle de voluntárias, 2,4-D, manejo, tiguera, Glycine max

Abstract - Volunteer soybean control is a fundamental requirement, mainly because of the rust inoculum disease present in these plants. Several herbicides are available that will control volunteer soybean in the vegetative growth stage, however farmers may delay application of control measures until the flowering growth stage, where the control can be more difficult With the introduction of 2,4-D tolerant soybean (DAS-444Ø6-6 - Enlist E3 ${ }^{\mathrm{TM}}$ ), other methods of chemical control need to be identified. The objective of this research was to determine the efficacy to control volunteer DAS-444Ø6-6 soybean using alternative herbicides. A field experiment was conducted in Cascavel-PR, using a randomized complete block design, with three replications. Herbicides treatments applied were halauxifen-methyl + pyroxsulam at $5 \mathrm{~g}$ a.i. $\mathrm{ha}^{-1}+18 \mathrm{~g}$ ai ha ${ }^{-1}$, halauxifen-methyl + diclosulam at $5 \mathrm{~g}$ a.i. ha $\mathrm{h}^{-1}+25 \mathrm{~g}$ ai ha ${ }^{-1}$ and halauxifen-methyl $+2,4-\mathrm{D}$ at $5 \mathrm{~g}$ a.i. $\mathrm{ha}^{-1}+783 \mathrm{~g}$ ai ha ${ }^{-1}$, and the standard paraquat $400 \mathrm{~g}$ ai ha- ${ }^{-1}$. The treatments were applied post emergence to soybean at the $R_{2}$ growth stage. Paraquat and the treatment formulations containing halauxifen-methyl provided effective control of volunteer DAS-444Ø6-6 soybean sprayed at advanced growth stage $\left(\mathrm{R}_{2}\right)$. Keywords: soybean volunteer control, 2,4-D, management, volunteer soybeans, Glycine max

Recebido: Junho 01, 2017. Aceito: Setembro 05, 2017.

${ }^{1}$ Dow AgroSciences Industrial Ltda, Av. Nações Unidas, 14.171, CEP 04730-090, São Paulo, SP, Brasil.

E-mail: 1szobiole@dow.com 


\section{Introdução}

O 2,4-D (ácido diclorofenóxiacético) foi o primeiro herbicida orgânico e seletivo desenvolvido na agricultura e permanece sendo utilizado há mais de 7 décadas e em mais de 100 países. Na última década, a descoberta de genes em bactérias de solo e sua introdução em plantas possibilitou o desenvolvimento de culturas transgênicas com tolerância ao 2,4-D (Griffin et al., 2013).

A soja DAS-444Ø6-6 (Enlist E3 ${ }^{\mathrm{TM}}$ ) será o primeiro transgênico comercial tolerante a este herbicida no Brasil, pois possui a enzima SdpA ou AAD-12 (aryloxyalkanoate dioxygenase-12), que catalisa a conversão do 2,4-D em 2,4-diclorofenol (2,4-DCP) e glioxilato (Wright et al., 2010). A introdução da soja tolerante ao 2,4-D irá ampliar a janela de uso do herbicida, podendo ser utilizado também na condição de pós-emergência da cultura (Robinson et al., 2015).

O uso de cultivares de soja tolerante ao glyphosate gerou profundas mudanças no controle químico da soja voluntária, também conhecida como tiguera ou guaxa, já que o referido herbicida deixou de ser utilizado como alternativa de controle nessa situação (Dan et al., 2009). Da mesma forma, a introdução da soja DAS-444Ø6-6 causará mudanças no controle químico da soja voluntária, já que o 2,4-D deixará de ser uma das alternativas para o seu controle, sendo necessário a utilização de outros herbicidas visando ao manejo destas plantas voluntárias.

O controle de soja voluntária agravou-se nos últimos anos com a regulamentação do vazio sanitário, cuja implementação tem sido realizada por meio de leis estaduais (Lima et al., 2011a). $O$ vazio sanitário estabelece a eliminação de plantas que podem hospedar doenças da cultura da soja, sobretudo a ferrugem asiática (Phakopsora pachyrhizi) (Terasawa et al., 2009). Deste modo, o conhecimento das alternativas de controle químico de plantas voluntárias de soja DAS-444Ø6-6 será essencial para a adoção comercial desta tecnologia no Brasil.

O halauxifen-methyl (Arylex ${ }^{\mathrm{TM}}$ ) é um novo herbicida mimetizador de auxinas desenvolvido pela Dow AgroSciences Industrial Ltda., e que pertence ao grupo químico dos arylpicolinatos (EFSA, 2015). O halauxifen-methyl possui elevada eficácia de controle sobre diversas plantas daninhas latifoliadas (Braz et al., 2017). Ademais, é altamente seletivo para diversos cereais de inverno e verão e possui baixo efeito residual devido à rápida degradação no solo e na palha (EFSA, 2015; Epp, 2016). No Brasil, este ingrediente ativo será registrado em mistura pré-formulada com outros herbicidas, como 2,4-D, diclosulam e pyroxsulam, de modo a atender diferentes segmentos da agricultura.

O presente trabalho teve como objetivo avaliar associações herbicidas contendo o ingrediente ativo halauxifen-methyl no controle da soja voluntária DAS-444Ø6-6 (Enlist E3 ${ }^{\mathrm{TM}}$ ), com aplicação sob plantas em estádio avançado de desenvolvimento.

\section{Material e métodos}

$\mathrm{O}$ ensaio foi conduzido em condições de campo, durante o período de outubro a dezembro de 2016, no município de Cascavel, Paraná ( $24^{\circ} 53^{\prime} 15^{\prime \prime}$ S; 53 32'60” N). O clima predominante na região, de acordo com a classificação de Köppen, é o do tipo Cfa - úmido mesotérmico. A precipitação média anual está entre 1.600 e $1.800 \mathrm{~mm}$ (IAPAR, 2014). O solo é da classe Nitossolo Vermelho eutroférrico, sem a presença de alumínio, apresentando textura argilosa (702 $\mathrm{g} \mathrm{kg}^{-1}$ de argila, $164 \mathrm{~g} \mathrm{~kg}^{-1}$ de silte, $134 \mathrm{~g} \mathrm{~kg}^{-1}$ de areia) e relevo semiondulado. Os dados de precipitação da localidade durante a condução do ensaio estão apresentados na Figura 1.

$\mathrm{O}$ delineamento experimental adotado foi o de blocos ao acaso, com três repetições. Os tratamentos constaram de três misturas formuladas contendo o ingrediente ativo halauxifen-methyl: halauxifen-methyl + pyroxsulam $\left(5+18\right.$ g i.a. ha $\left.{ }^{-1}\right)$, halauxifen-methyl + diclosulam $\left(5+25\right.$ g i.a. ha $\left.{ }^{-1}\right)$ e halauxifen-methyl+2,4-D $\left(5+783\right.$ g i.a. ha $\left.{ }^{-1}\right)$, acrescidos de tratamento com aplicação do herbicida paraquat $\left(400 \mathrm{~g}\right.$ i.a. ha $\left.{ }^{-1}\right)$ e testemunha sem aplicação, conforme descrito na Tabela 1. 


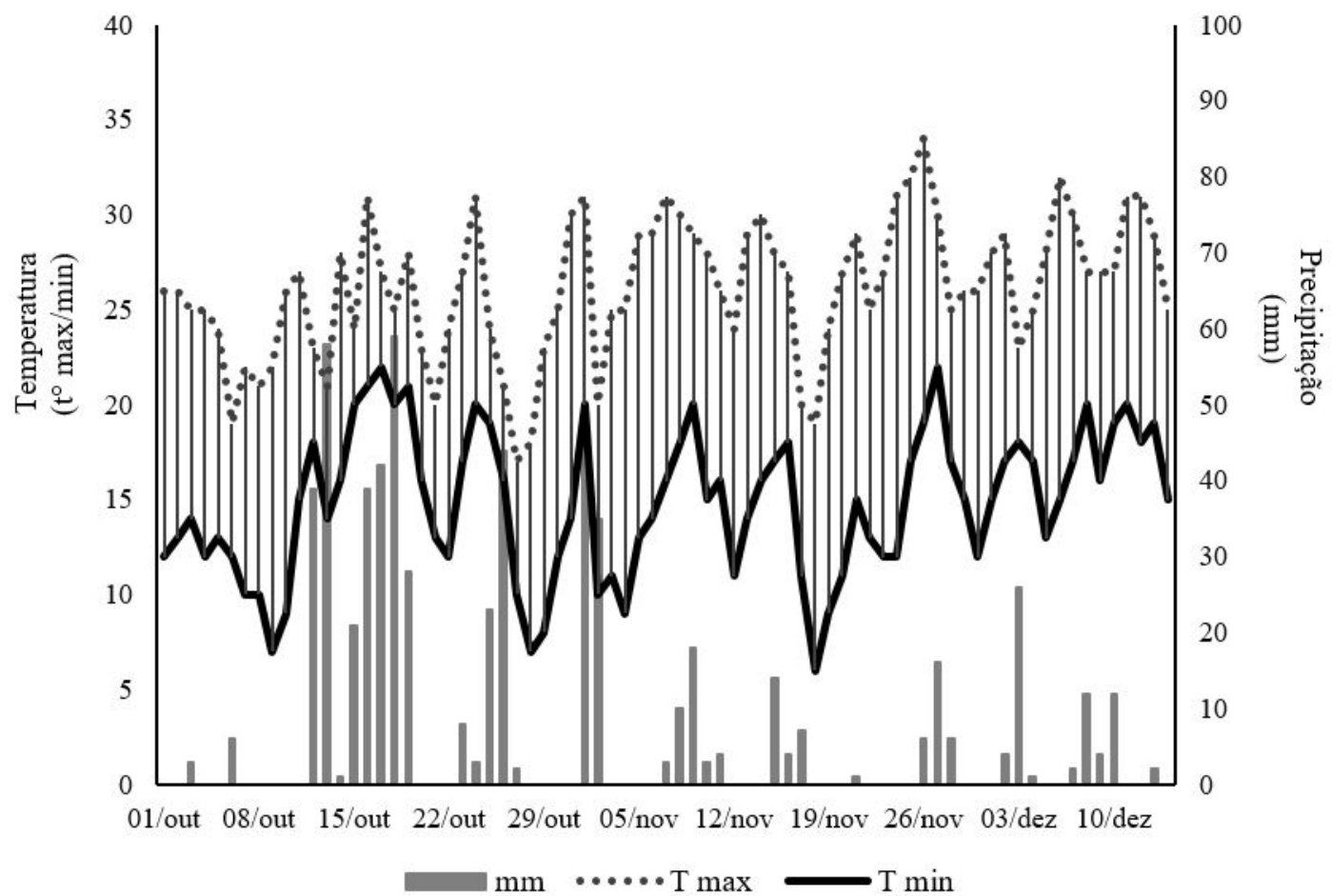

Figura 1. Precipitação pluvial diária e temperatura mínima e máxima diárias durante a condução do ensaio. Cascavel (PR), 2016.

Tabela 1. Tratamentos herbicidas testados e suas respectivas doses para o controle da soja voluntária DAS-444Ø6-6 em estádio $\mathrm{R}_{2}$. Cascavel (PR), 2016.

\begin{tabular}{|c|c|c|}
\hline \multirow{2}{*}{ Tratamento } & \multicolumn{2}{|c|}{ Doses } \\
\hline & g i.a. ha h $^{-1}$ & g ou L p.c. ha $^{-1}$ \\
\hline Halauxifen-methyl + pyroxsulam ${ }^{1 /}$ & $5+18$ & 0,7 \\
\hline Halauxifen-methyl + diclosulam ${ }^{1 /}$ & $5+25$ & 44 \\
\hline Halauxifen-methyl + 2,4-D ${ }^{1 /}$ & $5+783$ & 1,25 \\
\hline Paraquat $^{2 /}$ & 400 & 2 \\
\hline Testemunha sem aplicação & - & - \\
\hline
\end{tabular}

${ }^{1 /}$ Tratamento aplicado em associação com óleo éster metilado de soja GF-2968-MSO na dose de 1 L p.c. ha ${ }^{1}$ $\left(720\right.$ g i.a. ha $\left.{ }^{-1}\right) ;{ }^{2 /}$ Tratamento aplicado em conjunto com Agral $^{\circledR}$ a $0,1 \% \mathrm{v} \mathrm{v}^{-1}$.

As parcelas apresentavam dimensões de $3 \times 5 \mathrm{~m}$ (área bruta $=15 \mathrm{~m}^{2}$ ), com área útil de $10 \mathrm{~m}^{2}$, desconsiderando a bordadura de $0,5 \mathrm{~m}$.

A linhagem de soja utilizada no experimento foi advinda do programa de melhoramento genético da DONMARIO Sementes, sendo a mesma adaptada às condições climáticas da região Sul do Brasil, contendo o evento biotecnológico DAS-444Ø6-6 (Enlist E3 ${ }^{\mathrm{TM}}$ ) (Dow AgroSciences LLC, Indianapolis, IN). A aplicação dos tratamentos herbicidas ocorreu no dia $28 / 11 / 2016$, quando a soja voluntária DAS-444Ø6-6 atingiu o estádio fenológico de $\mathrm{R}_{2}$ (pleno florescimento da cultura), de acordo com a escala de Fehr e Caviness (1977).

A aplicação foi realizada utilizando pulverizador costal de pressão constante à base de $\mathrm{CO}_{2}$, equipado com barra de quatro pontas tipo leque AIXR 110.015, espaçadas entre si de $0,5 \mathrm{~m}$ (faixa de aplicação de $2 \mathrm{~m}$ ), sob pressão de sob pressão de $2 \mathrm{kgf} \mathrm{cm}^{-2}$. Estas condições de aplicação proporcionaram volume de calda equivalente a $100 \mathrm{~L} \mathrm{ha}^{-1}$. No momento da aplicação, o solo 
encontrava-se úmido, com temperatura do ar de $26^{\circ} \mathrm{C}$, a umidade relativa do ar em $64 \%$ e ventos de $1,9 \mathrm{~km} \mathrm{~h}^{-1}$. A população de plantas de soja voluntária era de $36 \mathrm{~m}^{-2}$.

A avaliação de controle da soja voluntária DAS-444Ø6-6 foi realizada aos 3, 7, 14 e 21 dias após aplicação (DAA), de acordo com a escala visual de $0-100 \%$, onde $0 \%$ significa ausência de sintomas e $100 \%$ morte total das plantas (SBCPD, 1995). Como padrão de comparação para avaliação dos tratamentos, utilizou-se como referência as plantas de soja que não receberam a aplicação dos tratamentos (testemunha sem aplicação). Além das avaliações de controle, na última avaliação, foram coletadas 10 plantas de soja remanescentes na parcela, dentro da área útil, a fim de avaliar a massa das plantas verdes, normalizando-se os valores obtidos em valor percentual em relação à testemunha não tratada.

Os dados foram submetidos à análise de variância pelo teste $\mathrm{F}$ e as médias comparadas pelo teste de Tukey, a 1\% de probabilidade.

\section{Resultados e discussão}

Foi observada diferença no controle da soja voluntária DAS-444Ø6-6 com os diferentes tratamentos testados (Tabela 2). Aos 7 DAA, os tratamentos contendo halauxifen-methyl não propiciaram controle satisfatório, enquanto que a eficácia do paraquat foi de 90\%. Devido ao seu mecanismo de ação, mimetizador de auxinas, o halauxifen-methyl proporcionou, inicialmente, apenas sintomas parecidos aos do 2,4-D, como epinastia foliar, deformação e necrose.

Em geral, as associações de halauxifen-methyl com 2,4-D e diclosulam apresentaram relativamente maiores valores de controle, do que o tratamento com halauxifen-methyl + pyroxsulam. Como o herbicida diclosulam também é absorvido via foliar (Grey e Prostko, 2015), pode ter contribuído para a maior eficácia em relação ao tratamento com pyroxsulam, uma vez que a seletividade das plantas aos herbicidas inibidores da acetolactato sintase (ALS) se relaciona, principalmente, à taxa de metabolização diferenciada entre as espécies e à velocidade de absorção e translocação desses produtos (Brown, 1990).

O controle pela aplicação do herbicida paraquat, avaliado aos 3 e 7 DAA, evidenciou rápida ação desse herbicida, caracterizada pela necrose foliar (Tabela 2). Essa rápida ação é devido ao seu mecanismo de ação ser a inibição do transporte de elétrons a partir do fotossistema I, ao qual rapidamente promove a formação de radicais livres, que rapidamente destroem as membranas tanto de organelas como da própria plasmalema (Summers, 1980). Dan et al. (2009) em trabalho realizado em ambiente controlado, também

Tabela 2. Percentual de controle e peso da massa verde da soja voluntária DAS-444Ø6-6 (AAD12) pelos tratamentos utilizados em pós-emergência. Cascavel (PR), 2016.

\begin{tabular}{|c|c|c|c|c|c|c|}
\hline \multirow{2}{*}{ Tratamentos } & \multirow{2}{*}{$\begin{array}{c}\text { Doses } \\
\left(\mathrm{g}^{\mathrm{i} . a .} \text { ha }^{-1}\right)\end{array}$} & \multicolumn{4}{|c|}{$\%$ Controle } & \multirow{2}{*}{$\begin{array}{l}\text { Peso (g) } \\
21 \text { DAA }\end{array}$} \\
\hline & & 3 DAA & 7 DAA & 14 DAA & 21 DAA & \\
\hline Halauxifen-methyl + pyroxsulam ${ }^{1 /}$ & $5+18$ & $18,0 \mathrm{~b}$ & $42,0 \mathrm{~b}$ & $67,0 \mathrm{c}$ & $91,0 \mathrm{~b}$ & $299,0 \mathrm{~b}$ \\
\hline Halauxifen-methyl + diclosulam ${ }^{1 /}$ & $5+25$ & $27,0 \mathrm{ab}$ & $71,0 \mathrm{a}$ & $90,0 \mathrm{~b}$ & 99,0 a & $167,0 \mathrm{c}$ \\
\hline Halauxifen-methyl + 2,4-D ${ }^{1 /}$ & $5+783$ & $35,0 \mathrm{ab}$ & $76,0 \mathrm{a}$ & $92,0 \mathrm{~b}$ & $100,0 \mathrm{a}$ & $128,0 \mathrm{c}$ \\
\hline Paraquat $^{2 /}$ & 400 & $53,0 \mathrm{a}$ & $90,0 \mathrm{a}$ & $99,0 \mathrm{a}$ & $100,0 \mathrm{a}$ & $23,0 \mathrm{~d}$ \\
\hline Testemunha sem aplicação & - & 0,0 & 0,0 & 0,0 & 0,0 & $604,0 \mathrm{a}$ \\
\hline $\mathrm{F}$ & & $16,89 *$ & $22,73 *$ & $9,16^{*}$ & $13,35^{*}$ & $0,15^{*}$ \\
\hline DMS & & 5,99 & 5,60 & 2,25 & 3,04 & 0,05 \\
\hline $\mathrm{CV}$ & & 22,45 & 8,02 & 3,13 & 3,60 & 2,46 \\
\hline
\end{tabular}

${ }^{1 /}$ Tratamento aplicado em associação com óleo éster metilado de soja GF-2968-MSO na dose de 1 L p.c. ha ${ }^{-1}$ $\left(720\right.$ g i.a. ha $\left.{ }^{-1}\right) ;{ }^{2 /}$ Tratamento aplicado em conjunto com Agral ${ }^{\circledR}$ a $0,1 \% \mathrm{v} \mathrm{v}^{-1}$. $*$ Médias na mesma coluna seguidas da mesma letra não diferem entre si pelo teste de Tukey $(\mathrm{p}<0,01)$. 
observou controle de $97 \%$ quando aplicado paraquat a $400 \mathrm{~g}$ i.a. ha ${ }^{-1}$ na soja voluntária, porém em estádio $\mathrm{V}_{3}$.

Nas avaliações realizadas aos 14 e 21 DAA, foi observado evolução considerável no controle da soja voluntária DAS-444Ø6-6 utilizando as misturas formuladas contendo halauxifen-methyl (Tabela 2). De maneira geral, embora se tenham observado diferenças entre os tratamentos compostos pela mistura com halauxifen-methyl, os tratamentos com halauxifen-methyl + diclosulam e halauxifen-methyl + 2,4-D foram estatisticamente superiores ao tratamento halauxifen-methyl + pyroxsulam, fato este provavelmente relacionado à taxa de metabolização diferenciada (Brown, 1990).

Aos 21 DAA, todos os tratamentos com halauxifen-methyl apresentaram controle aceitável da soja voluntária, porém os tratamentos com halauxifen-methyl + diclosulam e halauxifen-methyl + 2,4-D não evidenciaram diferença em relação ao paraquat (Tabela 2). Em estudo similar realizado com a aplicação isolada de halauxifen-methyl, entre outros herbicidas, também se observou controle satisfatório (98\%) de plantas voluntárias de soja DAS-444Ø6-6 com o uso desta nova auxina sintética, quando aplicado no estádio $\mathrm{V}_{3}$ (Lucio et al., 2016).

Em relação à massa verde de plantas, observou-se que o tratamento com a associação entre halauxifen-methyl + pyroxsulam apresentou, estatisticamente, maior massa verde em relação às demais formulações. Este resultado provavelmente deu-se pela morte mais lenta das plantas de soja voluntária DAS-444Ø6-6, em relação aos demais tratamentos.

Da mesma forma, o tratamento com paraquat apresentou o menor peso de massa verde em relação aos demais, tal resultado decorre da rápida morte da planta já a partir da primeira semana após aplicação (Tabela 2). Resultado semelhantes foi obtido por Lima et al. (2011b), ao qual compararam a eficácia de herbicidas no controle da soja voluntária tolerante ao glifosato em diferentes estádios de desenvolvimento, e observaram que tratamento que apresentou menor massa de plantas foi aquele com paraquat + diuron $\left(600+300\right.$ g i.a. ha $\left.{ }^{-1}\right)$, o que ocorreu pelo rápido controle em relação aos demais tratamentos quando aplicados no estádio fenológico $\mathrm{R}_{2} \mathrm{da}$ soja voluntária.

A resposta do controle da soja voluntária pode sofrer interferência relacionada ao estádio fenológico das plantas, cuja aplicação de paraquat + diuron $\left(600+300 \mathrm{~g} \mathrm{ha}^{-1}\right)$ não proporcionou morte das plantas de soja quando aplicado no estádio $\mathrm{R}_{2}$, entretanto controlou eficientemente quando aplicado em $V_{2}$ ou $V_{6}$ (Lima et al., 2011b). Embora neste trabalho não se tenha avaliado o efeito de estádios de aplicação, os resultados demonstram que existem alternativas de controle químico a soja voluntária DAS-444Ø6-6, quando aplicado em estádio avançado de desenvolvimento, no caso estádio $\mathrm{R}_{2}$.

Os resultados obtidos neste trabalho demonstram que os herbicidas à base de halauxifen-methyl podem constituir-se em opções para o controle de plantas de soja voluntária DAS-444Ø6-6, tolerante ao 2,4-D. Ademais, herbicidas convencionais no controle de soja voluntária tolerante ou não ao herbicida glifosato, como atrazina, metsulfuron metílico, trifloxysulfuron e paraquat, entre outros, continuarão sendo opções para o manejo de plantas voluntárias da soja DAS-444Ø6-6 (Lucio et al., 2016). Com isso, o sojicultor poderá utilizar variedades de soja DAS-444Ø6-6 com diversas opções à sua disposição para o manejo de plantas voluntárias nas culturas subsequentes.

\section{Conclusão}

Os tratamentos contendo halauxifen-methyl, independentemente do herbicida aplicado em associação (pyroxsulam, diclosulam e 2,4-D), foram eficazes no controle da soja voluntária DAS-444Ø6-6 em estádio fenológico $\mathrm{R}_{2}$, bem como o tratamento composto pela aplicação de paraquat isolado. 


\section{Referências}

Braz, G.B.P.; Oliveira Júnior, R.S.; Zobiole, L.H.S.; Rubin, R.S.; Voglewede, C.; Constantin, J.; Takano, H.K. Sumatran fleabane (Conyza sumatrensis) control in no-tillage soybean with diclosulam plus halauxifen-methyl. Weed Technology, v.31, n.1, p.1-9, 2017.

Brown, H.M. Mode of action, crop selectivity, and soil relations of the sulfonylurea herbicides. Pesticide Science, v.29, n.3, p.263-281, 1990.

Dan, H.A.; Barroso, A.L.L.; Procópio, S.O.; Dan, L.G.M.; Oliveira Neto, A.M.; Guerra, N.; Braz, G.B.P. Controle Químico de plantas voluntárias de soja Roundup Ready. Revista Brasileira de Herbicidas, v.3, n.3, p.96-101, 2009.

EFSA-European Food Safety Authority. Conclusion on the peer review of the pesticide risk assessment of the active substance halauxifen-methyl (XDE729 methyl). Italy: EFSA, 2015. Disponível em: $<$ http:/www.efsa.europa.eu/sites/default/files/ scientific_output/files/main_documents/3913.pdf $>$. Acesso em: 10 jan. 2017.

Epp, J.B. The discovery of ArylexTM active and RinskorTM active: two novel auxin herbicides. Bioorganic \& Medicinal Chemistry, v.24, p.362371, 2016.

Fehr, W.R.; Caviness, C.E. Stages of soybean development. Ames: Iowa State University, 1977. $12 \mathrm{p}$.

Grey, T.L.; Prostko, E. Uptake, translocation, and dose response of postemergence applied diclosulam to bristly starbur (Acanthospermum hispidum). Peanut Science, v.1, p.23-29, 2015.

Griffin, S.L.; Godbey, J.A.; Oman, T.J.; Embrey, S.K.; Karnoup, A.; Kuppannan, K.; et al. Characterization of aryloxyalkanoate dioxygenase-12, a nonheme fe(ii)/á-ketoglutarate-dependent dioxygenase, expressed in transgenic soybean and Pseudomonas fluorescens. Journal of Agricultural and Food Chemistry, v.61, p.6589-6596, 2013.
IAPAR - Instituto Agronômico do Paraná. Cartas climáticas do Paraná: precipitação. Londrina: IAPAR, 2014. Disponível em: <http://www.iapar.br/ modules/conteudo/conteudo.php?conteudo $=595>$. Acesso em: 10 jan. 2017.

Lima, D.B.C.; Silva, A.G.; Procópio, S.O.; Barroso, A.L.L.; Dan, H.A.; Costa, E.B.; Pereira, A.J.B. Seleção de herbicidas para o controle de plantas voluntárias de soja resistentes ao glyphosate. Revista Brasileira de Herbicidas, v.1, p.1-12, 2011a.

Lima, D.B.C.; Silva, A.G.; Procópio, S.O.; Barroso, A.L.L.; Dan, H.A. Controle químico de plantas voluntárias de soja Roundup Ready ${ }^{\circledR}$ em diferentes estádios de desenvolvimento. Revista Caatinga, v.3, p.63-70, 2011 b.

Lucio, F.R.; Rossi, C.V.S.; Rosseto, J.; Manzoni, C.G.; Zobiole, L.H.S.; Rampazzo, P.E.; Cunha, L.C. Eficácia de herbicidas para controle de soja Enlist $^{\mathrm{TM}}$ E3 voluntária em cultivos subsequentes. In: Congresso Brasileiro da Ciência das Plantas Daninhas, 30., 2016, Curitiba. Anais... Curitiba: UFSC, 2016. p.282.

Robinson, A.P.; Davis, V.M.; Simpson, D.M.; Johnson, W.G. Response of aryloxyalkanoate dioxygenase-12 transformed soybean yield components to postemergence 2,4-D. Weed Science, v.63, p.242-247, 2015.

SBCPD - Sociedade Brasileira da Ciência das Plantas Daninhas. Procedimentos para instalação, avaliação e análise de experimentos com herbicidas. Londrina: SBCPD, 1995. 42p.

Summers, L.A. The bipyridinium herbicides. New York: Academy Press, 1980. 449p.

Terasawa, J.M.; Panobianco, M.; Possamai, E.; Koehler, H.A. Antecipação da colheita na qualidade fisiológica de sementes de soja. Bragantia, v.3, p.765-773, 2009.

Wright, T.R.; Shan, G.; Walsh, T.A.; Lira, J.M.; Cui, C.; Song, P.; et al. Robust crop resistance to broadleaf and grass herbicides provided by aryloxyalkanoate dioxygenase transgenes. Proceedings of the National Academy of Sciences of the United States of America, v.107, p.20240-20245, 2010. 